\title{
DEVELOPMENT OF BROADBAND LOW ACTUATION VOLTAGE RF MEM SWITCHES*
}

\author{
S. C. SHEN ${ }^{\dagger}$, D. BECHER, Z. FAN, D. CARUTH and MILTON FENG \\ Center for Compound Semiconductor Microelectronics, Department of Electrical and Computer \\ Engineering, University of Illinois at Urbana-Champaign, 208 N. Wright St., Urbana, \\ IL 61801-2355, USA
}

(Received 6 November 2001; In final form 3 December 2001)

\begin{abstract}
Low insertion loss, high isolation RF MEM switches have been thought of as one of the most attractive devices for space-based reconfigurable antenna and integrated circuit applications. Many RF MEMS switch topologies have been reported and they all show superior RF characteristics compared to semiconductor-based counterparts. At the University of Illinois, we developed state-of-the-art broadband low-voltage RF MEM switches using cantilever and hinged topologies. We demonstrated promising sub-10 volts operation for both switch topologies. The switches have an insertion loss of less than $0.1 \mathrm{~dB}$, and an isolation of better than $25 \mathrm{~dB}$ over the frequency range from 0.25 to $40 \mathrm{GHz}$. The RF Model of the MEM switch was also established. The low voltage RF MEM switches will provide a solution for low voltage and highly linear switching methods for the next generation of broadband RF, microwave, and millimeter-wave circuits.
\end{abstract}

\section{INTRODUCTION}

In recent years, success in the development of low-cost millimeter-wave integrated circuits (MMICs) in the compound semiconductor industry has lead to numerous business opportunities in the wireless communication area. Due to the portable nature of mobile communication systems, power consumption is a major concern. Aside from the stand-by leakage current and power added efficiency issues in active devices, the losses through passive components are problematic as well. With the increasing demand for high performance radio frequency (RF) circuits, micro-electromechanical-systems (MEMS)-based components have drawn much attention over the past few years [1-2]. These micro-machined components such as high-Q inductors [3], capacitors [4] and switches [5-9] have demonstrated excellent RF characteristics. They have been thought of as a competing technology for the next generation of low power communication systems.

The low insertion loss, high isolation RF MEM switch is one of the most attractive devices for space-based reconfigurable antenna and integrated circuit applications [1]. Compared to active-device-based switches, MEM switches have an insertion loss one order of magnitude lower than that of PIN diode switches. The lossy RF characteristics of semiconductor-based

\footnotetext{
* Submitted to Special issue of APEC in "Discrete Micro-Components for H.F. Applications".

${ }^{\dagger}$ Corresponding author. Tel.: 217-333-4054; Fax: 217-244-6375; E-mail: scshen@hsic.micro.uiuc.edu
} 
switches are major problems for current MMICs. On the other hand, MEM switches have demonstrated an insertion loss of less than $0.1 \mathrm{~dB}$ and isolation as high as $30 \mathrm{~dB}$ up to $40 \mathrm{GHz}$. They provide GaAs phase shifters, for example, with reduced insertion loss for switching between different line lengths or between high and low-pass filters [10-12]. As the loss of the phase shifter arrays decreases, fewer amplifiers are required in phased antenna arrays. Cost, weight, and heat dissipation problems can be drastically reduced. It is expected that RF MEM switches can potentially take the place of their semiconductor counterparts in RF switching applications that require a switching speed of only a few hundred microseconds.

Many RF MEMS switch topologies have been reported. Typically, they are categorized as rotary [2], cantilever [5-6], and membrane switches [7-9]. All of them have demonstrated excellent RF characteristics. However, most of them need very high actuation voltages (usually 30-50 volts), making these switches impractical for mobile wireless communication applications.

Much effort has gone into the search for low voltage RF MEM switches [13-16]. The low actuation voltage operation will facilitate the direct integration of MEM switches with current MMICs without adding clumsy voltage up-converter circuitry. Moreover, the extremely broadband RF characteristics allow the switches to be easily incorporated into circuits with minimal effort in matching network design. The bandwidth of RF circuits can be further broadened accordingly.

In this work, we will focus on the development of GaAs-based surface micro-machined switches. Specifically, we are interested in low-voltage switch development because of its compatibility with mobile communication systems. Two switch topologies with cantilever and hinged structures are fabricated and studied. The device structures and their operation principles will be described in section 2. In section 3, a low-temperature fabrication process for RF MEM switches will be presented. The device performance will be discussed in section 4. The best RF data of fabricated cantilever switches shows an insertion loss of $<0.1 \mathrm{~dB}$ and an isolation of higher than $23 \mathrm{~dB}$ up to $40 \mathrm{GHz}$. The actuation voltage is 10-23 volts with a switching speed of $47 \mu \mathrm{s}$. It has become apparent that cantilever structures will not suffice for faster switching speed and low voltage operation simultaneously due to fundamental mechanical limitation. To overcome this trade-off, we propose a novel "hinged" topology to address both low-voltage and high-speed requirements for RF MEM switches [16]. Our results show a negligible insertion loss of $<0.1 \mathrm{~dB}$ and a nearly constant isolation of $>25 \mathrm{~dB}$ up to $40 \mathrm{GHz}$ [17-18] with a promising low switching voltage operation of 9 volts. For circuit design purposes, a generic lumped-circuit MEM switch model for both on and off state are also developed to emulate the RF characteristics of MEM switches and to provide useful information on the device performance improvement.

\section{DEVICE STRUCTURES}

\subsection{Cantilever Switches}

Cantilever beams are the most commonly used structure for MEMS components. The beams can be actuated electrostatically, magnetically, or thermally. The deflected beams provide moving parts in MEMS components with a restoring force when it is de-actuated. Shown in Figure 1(a) is a schematic drawing of a shunt cantilever switch. The RF signals are guided by a coplanar waveguide (CPW) structure. A metal pad is supported by cantilever beams to hold it in the switch-up position. The metal pad is pulled down by an electrostatic force and bounces back to its up position when the actuation voltage is removed, as shown in Figure 1(b). 


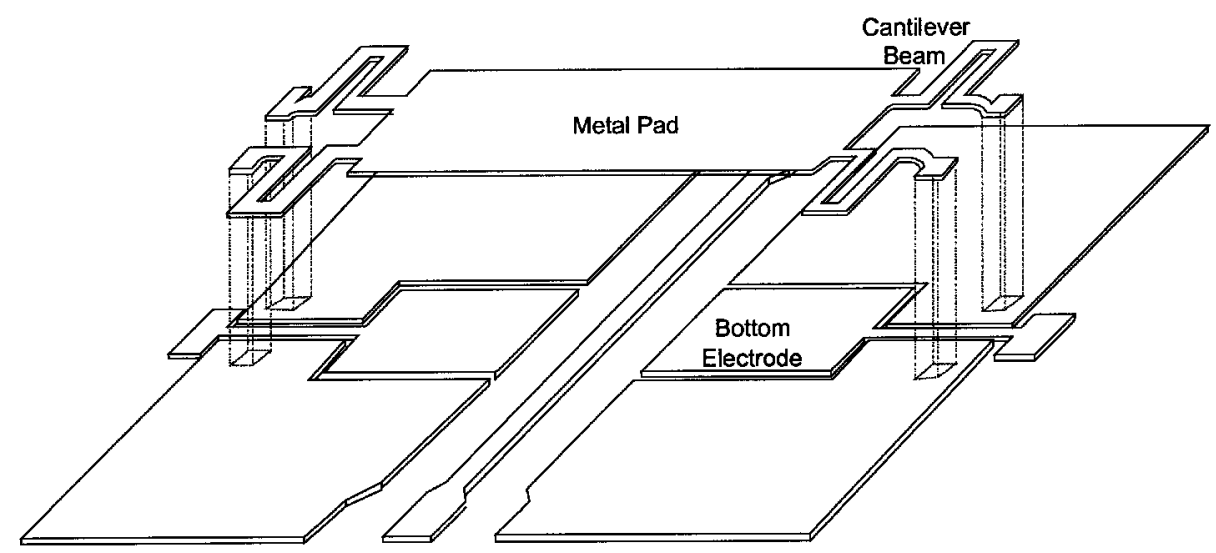

(a)

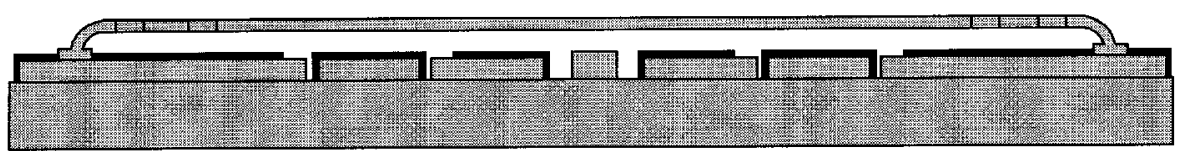

ON STATE

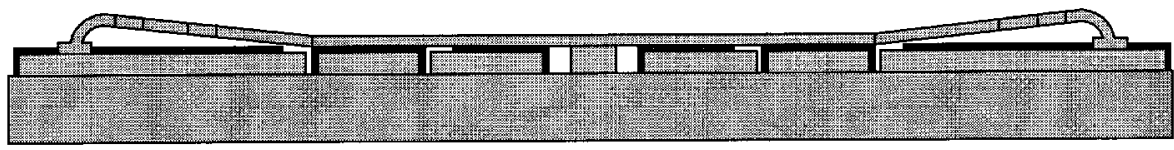

OFF STATE

(b)

FIGURE 1 A schematic drawing of a cantilever switch with (a) 3-D view and (b) cross-sectional view for on and off states, respectively.

In contrast with other cantilever switches, the actuation voltage is applied on a separated bottom electrode instead of the cantilever beams. This design scheme offers some advantages. First, the cantilever beams are electrically connected to the ground plane, separating the actuation signal from the RF signal. The intermodulation between actuation voltages and RF signals can be alleviated. This also leads to an extremely broadband RF characteristic. Second, it is equivalent to an air bridge in already-established MMIC fabrication processes.

The minimum actuation voltage of a cantilever switch, usually referred as 'pull-in voltage', can be expressed as [19]

$$
V_{p}=\sqrt{\frac{8 K g^{3}}{27 \varepsilon_{0} A}}
$$

where $K$ is the spring constant of the cantilever beams, $g$ is the gap between the metal pad and bottom electrodes, $\varepsilon_{0}$ is the permittivity of air, and $A$ is the area of the bottom electrode. In order to get lower voltage operation, the most effective way is to decrease the spring 
constant $K$. This can be achieved by selecting a low $K$ material and by utilizing a serpentine cantilever beam structure. The effective spring constant of a serpentine cantilever beam can be estimated as [13]:

$$
K=\frac{E w\left(t / L_{c}\right)^{3}}{N\left(1+L_{s} / L_{c}\left(\left(L_{s} / L_{c}\right)^{2}+12(1+v) /\left(1+(w / t)^{2}\right)\right)\right)}
$$

where $E$ and $v$ are the Young's modulus and Poisson's ratio of the cantilever beam; $w$ and $t$ are the width and thickness of the cantilever beam, respectively; $L_{s}$ is the length of the cantilever beam in each turn, and $L_{c}$ is the spacing between adjacent turns. $N$ is the total turns of the serpentine beam. Therefore, with a proper design of beam geometry and the use of low Young's modulus material such as gold, the spring constant can be effectively reduced and a lower actuation voltage can be achieved.

\subsection{Hinged Switch}

Another switch topology investigated in this work is the hinged switch. As shown in Figure 2(a), a conductive pad is inserted in between the top and bottom electrodes and hangs across the signal line and ground planes of a CPW. Brackets guide the pad as it moves up and down. The top and bottom electrodes facilitate the application of actuation voltages. The actuation voltage provides an electrostatic force to make the conductive pad move up and down in a single-pole-single-throw dual-electrode configuration.

When a voltage is applied on the bottom electrodes, the metal pad contacts the signal line and ground planes, as shown in Figure 2(b). The input RF signal from one port will short to the ground and no RF signal will flow through the other port. This corresponds to the switch 'off' state. When a voltage is applied to the top electrodes, the conductive pad is attracted upward. RF signals flow through the output port without much insertion loss. This corresponds to the switch 'on' state. The switching operation can therefore be realized by applying two out-of phase pulses on the top and bottom actuation electrodes. Ideally, the minimum electrostatic force required for actuation is equal to the sum of the weight and the air friction of the conductive pad. The minimum actuation voltage $V_{\min }$ required for the switch operation can be estimated by:

$$
V_{\min }=\sqrt{\frac{2\left(m g+F_{\text {friction }}\right)}{\varepsilon_{0} A}} d
$$

where $d$ is the spacing between the conductive pad the electrodes, $m g$ is the weight of the conductive pad, $F_{\text {friction }}$ is the frictional force, $A$ is the area of the actuation pad, and $\varepsilon_{0}$ is the permitivity of air. Assuming the metal pad is made of a $1 \mu \mathrm{m}$ gold film, the gap between electrodes is $3 \mu \mathrm{m}$, and the frictional force is negligible, the minimum actuation voltage is less than 2 volts.

The hinged switches provide several advantages. First, the high actuating voltage due to mechanical stress in MEM devices can be effectively reduced. Second, similar to the cantilever switch described in the previous paragraphs, the switch is electrically isolated from the actuating voltage, which will result in a wider bandwidth. Third, the device size can be relatively small to enable direct scaling to higher frequency applications. Finally, the switching speed is determined by the amount of electrostatic force exerted on the metal pad, rather than the spring restoring force. This will allow faster switching and less damping problems for hinged switches. 


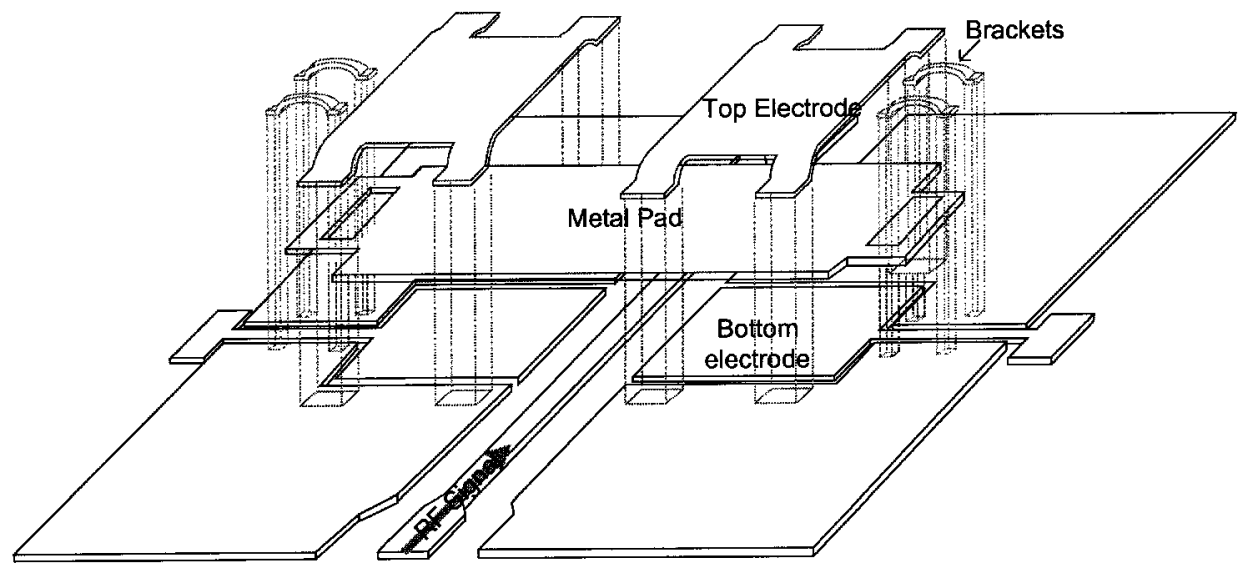

(a)

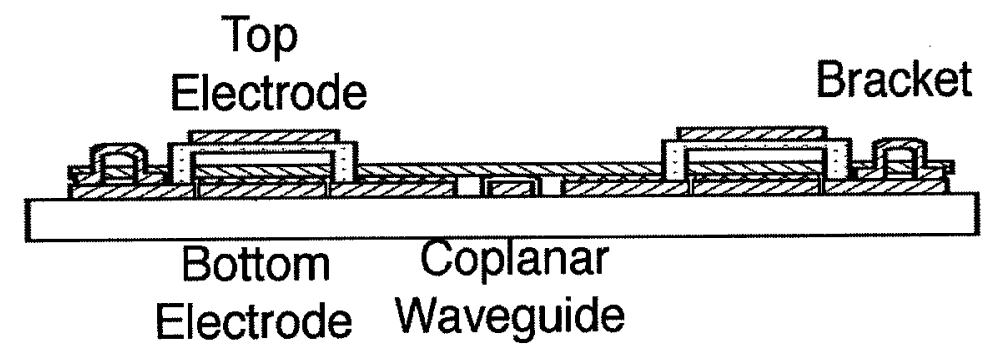

SWITCH ON

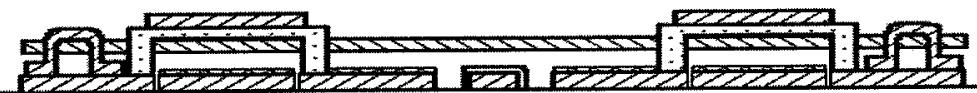

SWITCH OFF

(b)

FIGURE 2 A schematic drawing of a hinged RF MEM switch with (a) 3-D view and (b) cross-sectional side view for on and off states, respectively.

\section{FABRICATION PROCESSES}

We adapt the concept of surface micromachining on GaAs substrates for RF MEM switch fabrication processes. The process is developed to be compatible with standard CPWbased MMIC process.

The process flow of hinged switches is shown in Figure 3. The device fabrication is a 7 mask-layer process compatible with conventional GaAs MMIC process. First, a 1- $\mu$ m thick $\mathrm{Au}$ layer is evaporated and patterned to form the $\mathrm{CPW}$ and bottom electrodes. A layer of 


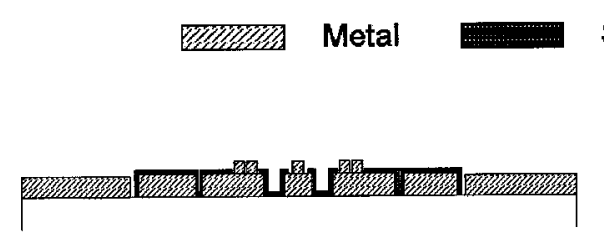

(a)

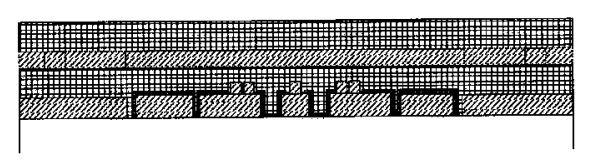

(c)

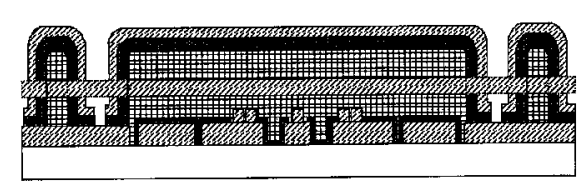

(e)

\section{SiNx Polyimide}

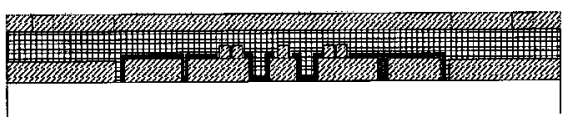

(b)

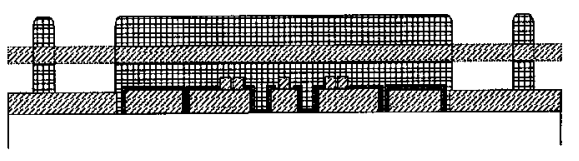

(d)

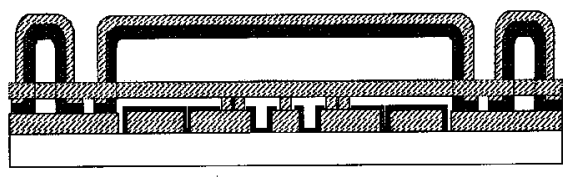

(f)

FIGURE 3 Hinged RF MEM switch process flow.

PECVD silicon nitride is deposited, followed by a via-hole etch (Fig. 3(a)). A Polyimide sacrificial layer is spun on and a $1 \mu \mathrm{m}$ thick $\mathrm{Au}$ layer is evaporated to form the conductive pad (Fig. 3(b)). A second polyimide layer is spun on (Fig. 3(c)) and is patterned for top electrode support posts (Fig. 3(d)). A low-temperature PECVD silicon nitride layer is deposited followed by an Au deposition to form top electrodes (Fig. 3(e)). The silicon nitride layer is then etched in an RIE system and sacrificial layers are removed by wet etch. Finally, the devices are released using a carbon-dioxide supercritical drying technique [20] (Fig. 3(f).)

Gold is chosen for the conductive pad metal in this study because of its low material stress. This leads to less warping of the conductive pad, and results in smaller parasitic capacitances in the switch. As a result, the device will have a lower insertion loss at switch on state.

The fabrication process of cantilever switches is similar to the hinged process. The sacrificial layer is released after the metal pad is fabricated and no top electrode is required, i.e., the process steps from Figures 3(c)-3(e) are skipped for cantilever switch fabrication. The resulting process steps are exactly the same as common-practice air bridge MMIC fabrication processes.

\section{RESULTS AND DISCUSSIONS}

The RF performance of fabricated switches is characterized by a s-parameter measurement. The s-parameters are measured using an HP8510C vector network analyzer and $150 \mu \mathrm{m}$ pitch RF coplanar probes. An on-wafer calibration scheme is adopted to eliminate pad parasitics. The calibration standards are built on a semi-insulating GaAs substrate with short, open, load, and through (SOLT) patterns. The pads effectively become a part of the measurement system 
after the on-wafer calibration, and the measured data are the s-parameters of the two-port located between the pads [21].

Device parameters extracted from s-parameter measurements are modeled in a HP Microwave Design System (MDS). The measured results are de-embedded using a CPW model developed by Shimon [22]. The device is decomposed into 2 sections of CPW and the intrinsic switch portion. The de-embedded data is used for the modeling of an "intrinsic" switch for circuit design purposes. A generic lumped-circuit model for both 'on' and 'off' states is shown in Figure 4 . The lumped circuit model simulates the finite length transmission line embedded in the device and the core portion of a shunt switch. $R_{11}, R_{12}, L_{11}$, and $L_{12}$ account for the resistance and the inductance of the finite length transmission line; $C_{p}$ is the parasitic capacitance

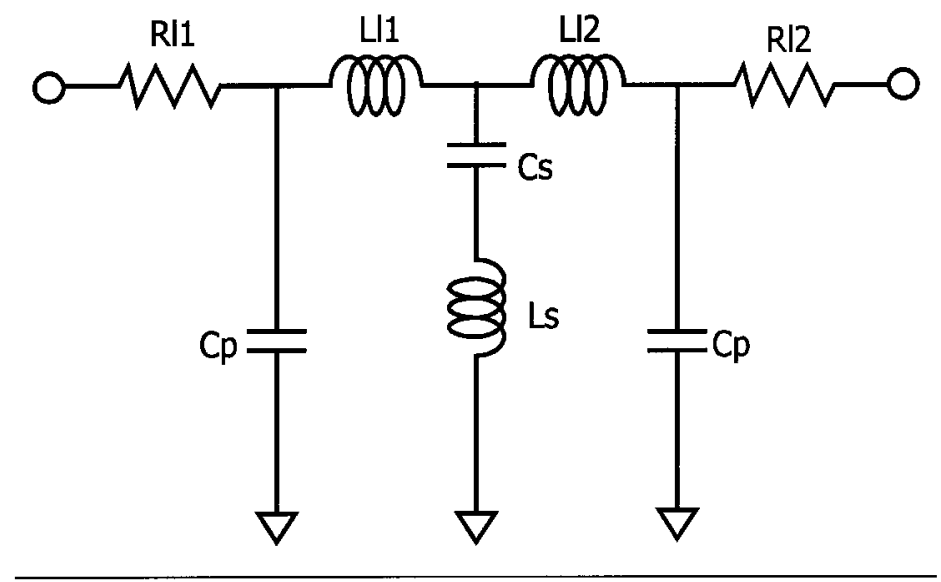

(a)

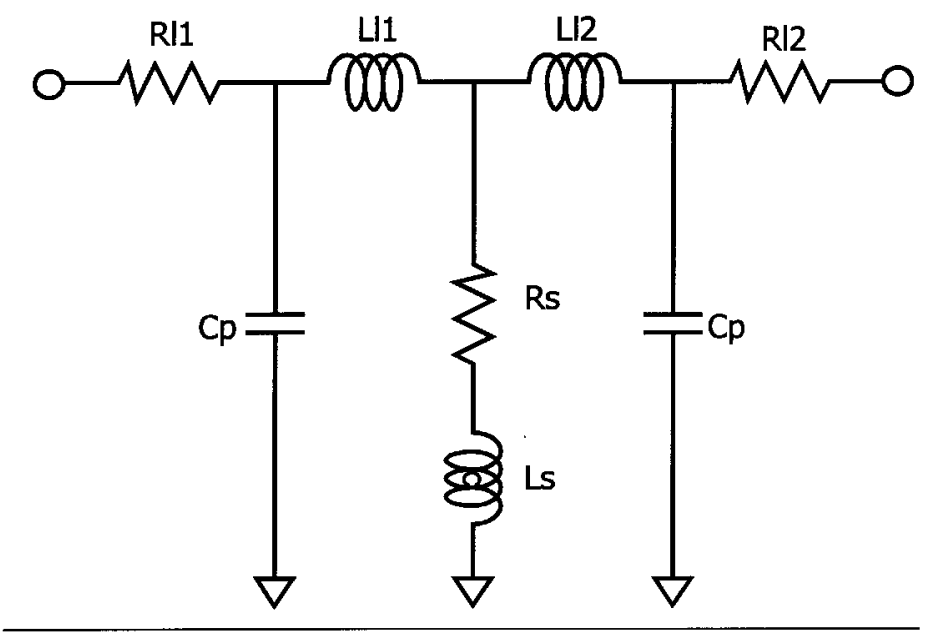

(b)

FIGURE 4 A generic equivalent circuit model of a shunt RF MEM switch for (a) on state and (b) off state. 
between the metal plate and the coplanar structure due to non-uniform metal contact. The core portion of the switch is essentially a resistance $R_{s}$ for the switch off state and a capacitance $C_{s}$ for the switch on state. $L_{S}$ accounts for the inductance of the suspended metal pad.

\subsection{Cantilever Switches}

A fabricated cantilever switch is shown in Figure 5. The metal pad along with four meandered cantilevers is made of a 1- $\mu$ m thick e-beam evaporated gold. Each cantilever has a dimension of $250 \mu \mathrm{m}$ in length and $8 \mu \mathrm{m}$ in width. The metal pad size is $80 \times 160 \mu \mathrm{m}^{2}$ and the bottom electrode size is $80 \times 85 \mu \mathrm{m}^{2}$ on each side of the center conductor of a CPW structure. The switch has a minimum switching voltage of 13 volts. The relatively low actuation voltage is a result of the serpentine cantilever structure and reduced material stress from using gold as the pad metal.

The RF performance is shown in Figure 6. For the switch on state, the switch has an insertion loss of less than $0.2 \mathrm{~dB}$ with a return loss of less than $30 \mathrm{~dB}$ at $40 \mathrm{GHz}$. For the switch off state, the switch shows an isolation of $23 \mathrm{~dB}$ at $20 \mathrm{GHz}$ and of $19 \mathrm{~dB}$ at $40 \mathrm{GHz}$.

Shown in Figure 7 is the measured switching speed of a cantilever switch. The control signal of $5 \mathrm{kHz}$ square waves is shown at the top trace of the oscilloscope. The corresponding drive signal is a square wave with an amplitude of 15 volts and is out of phase with the control signal. The modulated $1 \mathrm{MHz} \mathrm{HF}$ signal is shown at the bottom trace. The modulated signal shows a time delay of $47 \mu \mathrm{s}$ from the switching-on to the switching-off state. This time delay is defined as the switching speed of a switch. It accounts for the time needed for the metal pad to move from "switch-up" position to "switch-down" position.

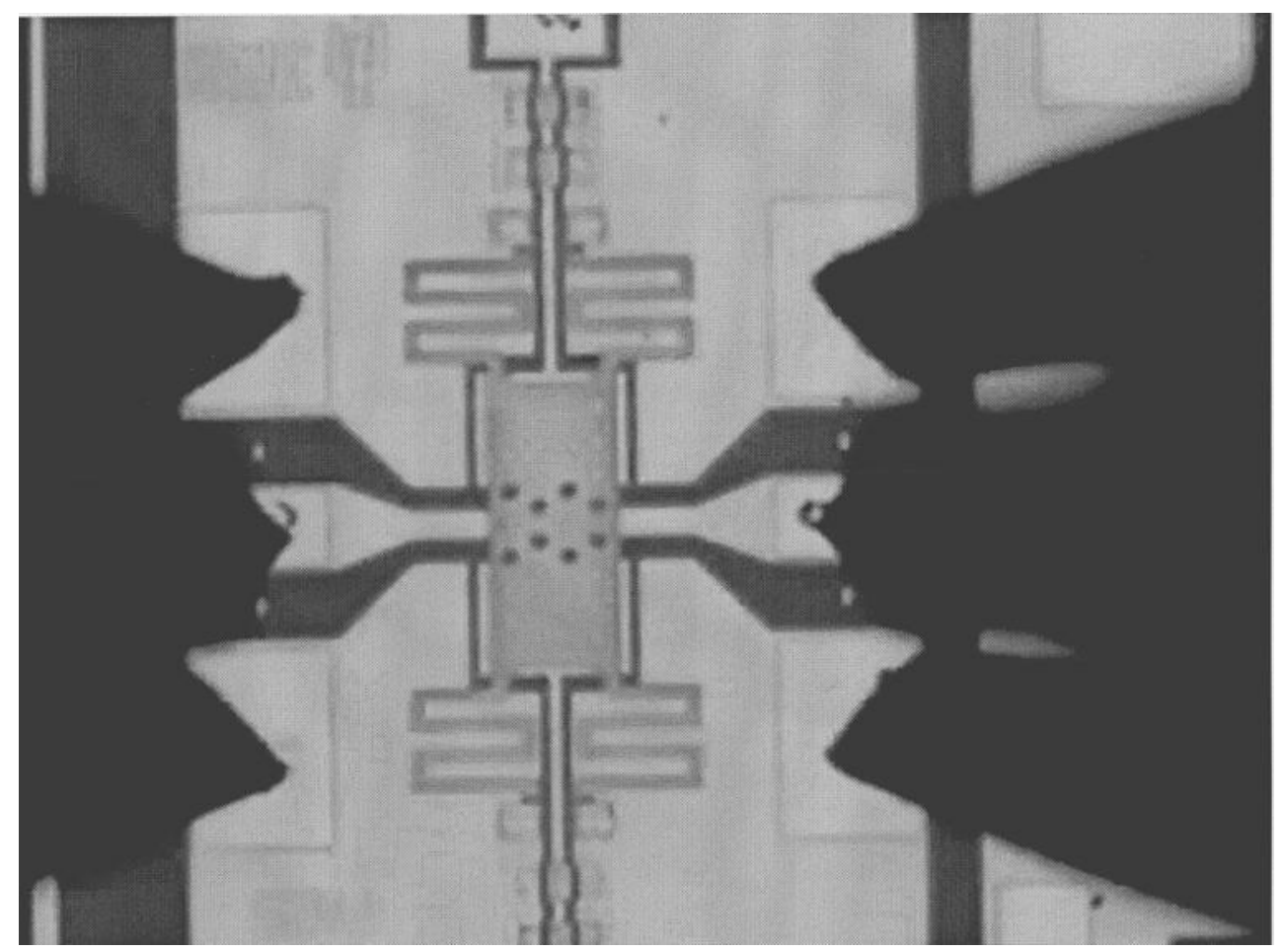

FIGURE 5 A microscope picture of a fabricated cantilever switch. 


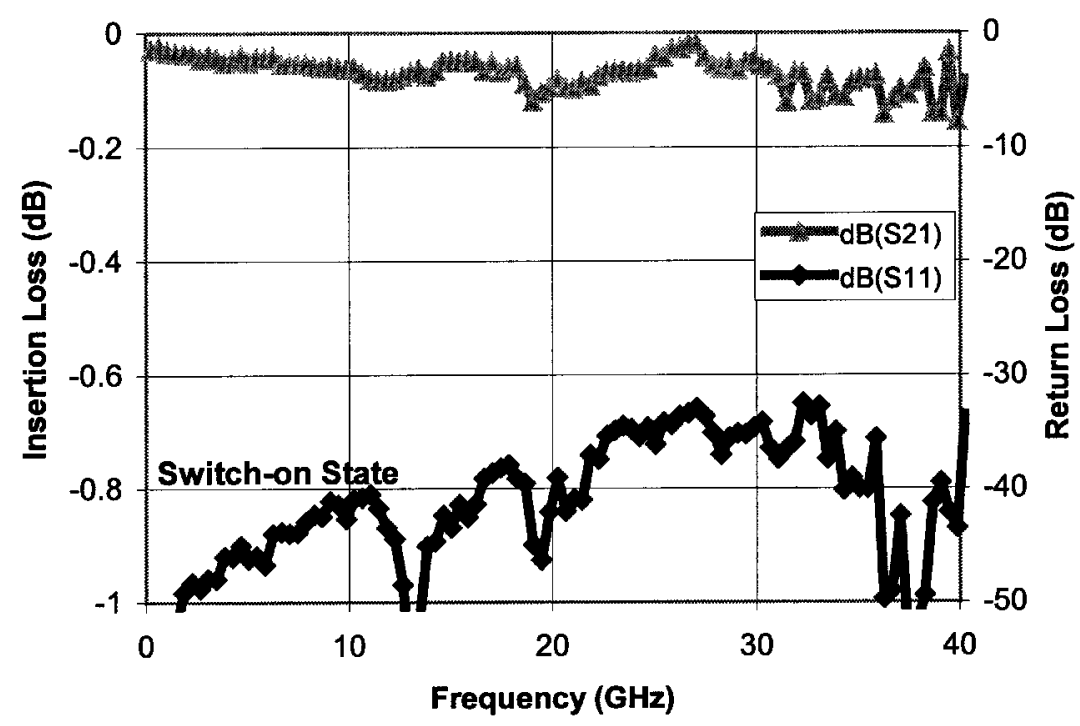

(a)

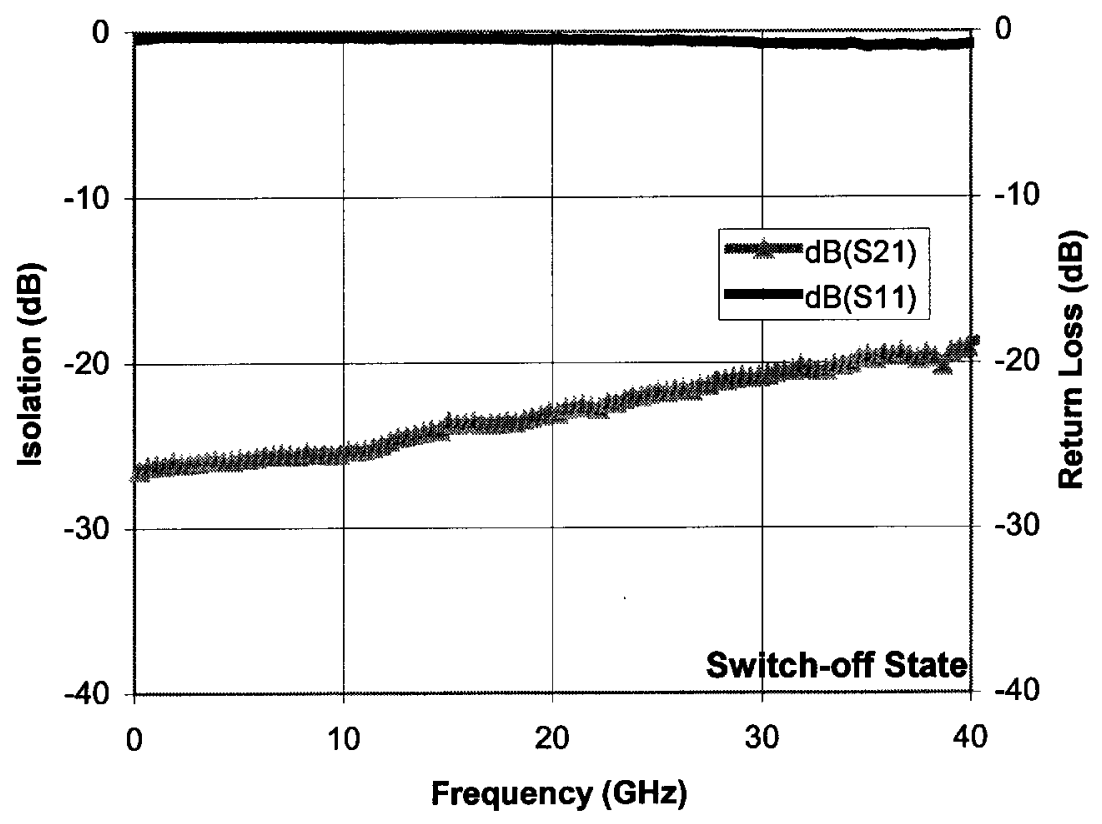

(b)

FIGURE 6 Measured RF performance for a cantilever switches for (a) switch-on state and (b) switch-off state. 


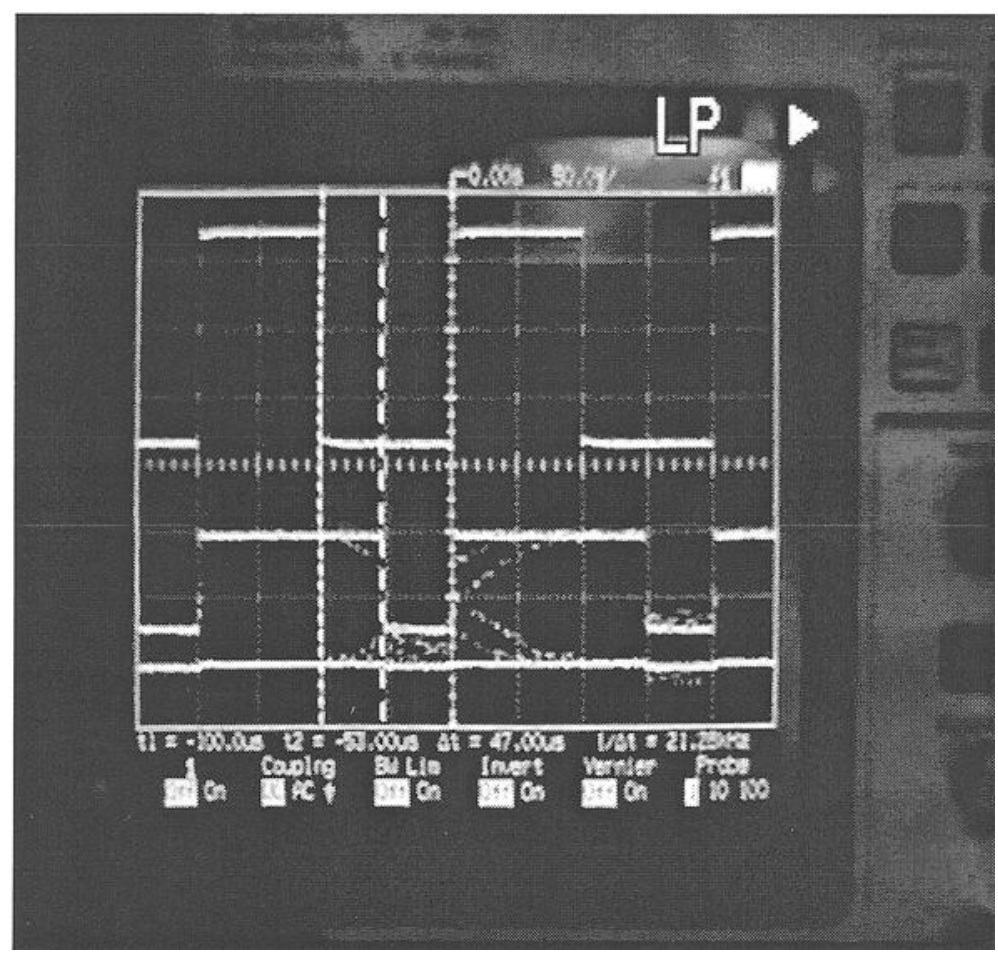

FIGURE 7 A switching speed measurement of a cantilever switch. The control signal is shown at the top trace and the modulated HF signal is shown at the bottom trace.

Listed in Table I is a summary of measurement results for cantilever switch with various device dimensions specified in Figure 8. The results show that the beam width and the total length of cantilever beams are two dominant factors for the actuation voltage of electrodes with similar pad size. In order to reduce the actuation voltage, it might be helpful to make a narrower beam and to stretch the beam length. The data show that a minimum actuation voltage of 9.5 volts can be achieved for devices with $L_{c}$ of $360 \mu \mathrm{m}$ and $W_{c}$ of $8 \mu \mathrm{m}$ with an isolation of $17 \mathrm{~dB}$ at $40 \mathrm{GHz}$. The relatively low isolation is attributed to the series inductance of the meandered serpentine beams at the switch down state.

The equivalent circuits are extracted using the scheme described in the previous section. A smith chart of the modeled parameter versus the measured data is shown in Figure 9. From the modeled parameters for the switch off state, we found that shunt resistance $R_{S}$ and inductance $L_{s}$ are major parameters need to be optimized for improved isolation. $R_{S}$ is related to the contact resistance of the switch between the metal pad and the center

TABLE I Measurement Results for Cantilever Switch with Different Dimensions Specified at Figure 8. The Isolation and Insertion Loss are Measured at $40 \mathrm{GHz}$.

\begin{tabular}{lccccccc}
\hline$W_{s}(\mu \mathrm{m})$ & $W_{p}(\mu \mathrm{m})$ & $L_{p}(\mu \mathrm{m})$ & $L_{c}(\mu \mathrm{m})$ & $W_{c}(\mu \mathrm{m})$ & $\begin{array}{c}\text { Actuation } \\
\text { voltage }(V)\end{array}$ & $\begin{array}{c}\text { Isolation } \\
(d B)\end{array}$ & $\begin{array}{c}\text { Insertion } \\
\text { loss }(d B)\end{array}$ \\
\hline 20 & 80 & 85 & 360 & 8 & 9.5 & 17 & 0.2 \\
20 & 80 & 85 & 250 & 8 & 13 & 19.5 & 0.2 \\
20 & 100 & 85 & 150 & 10 & 23 & 23 & 0.1 \\
20 & 100 & 85 & 360 & 10 & 19.5 & 20 & 0.05 \\
\hline
\end{tabular}




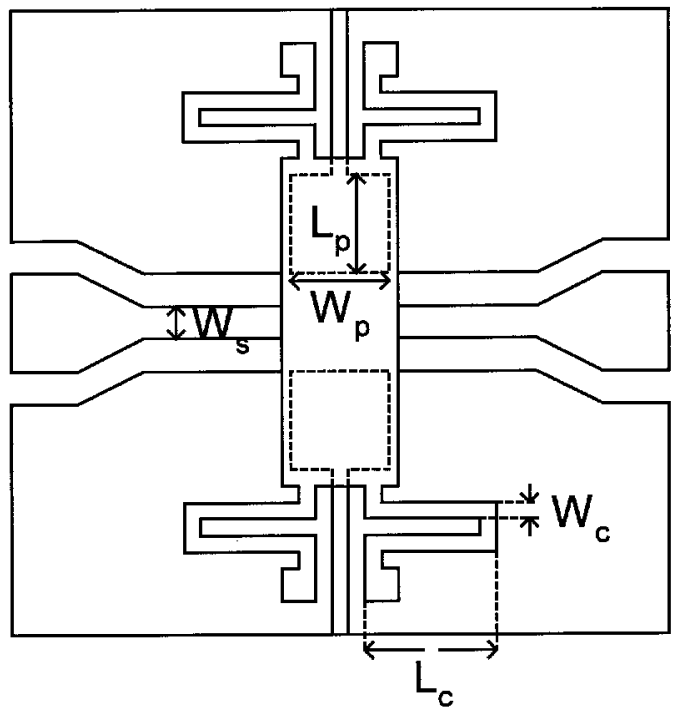

FIGURE 8 A schematic drawing that shows the dimension pecification of cantilever switches. $W_{p}$ and $L_{p}$ specify the dimension of bottom electrode pads, $W_{s}$ is the width of the center conductors of the CPW structure, $W_{c}$ is the width of the serpentine cantilever, and $L_{c}$ is the total length of the serpentine cantilever.

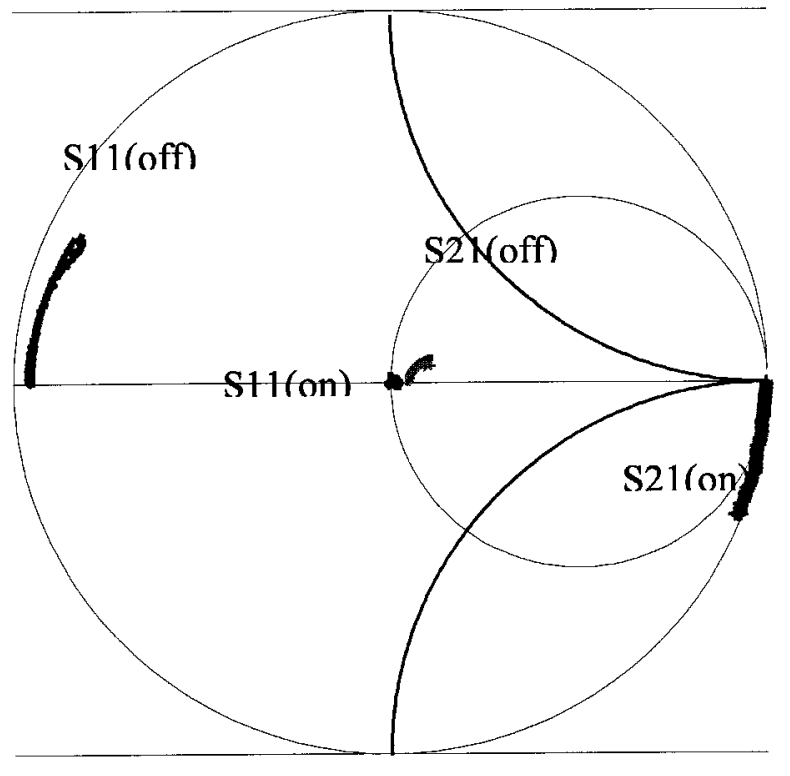

\begin{tabular}{|c|c|c|c|c|c|c|c|c|}
\hline State & $\begin{array}{c}\mathrm{R}_{\mathrm{L} 1} \\
(\Omega)\end{array}$ & $\begin{array}{c}\mathrm{L}_{\mathrm{L} 1} \\
(\mathrm{pH})\end{array}$ & $\begin{array}{c}\mathrm{R}_{\mathrm{L} 2} \\
(\Omega)\end{array}$ & $\begin{array}{c}\mathrm{L}_{\mathrm{L} 2} \\
(\mathrm{pH})\end{array}$ & $\begin{array}{c}\mathrm{C}_{\mathrm{p}} \\
(\mathrm{fF})\end{array}$ & $\begin{array}{c}\mathrm{L}_{\mathrm{s}} \\
(\mathrm{pH})\end{array}$ & $\begin{array}{c}\mathrm{C}_{\mathrm{s}} \\
(\mathrm{fF})\end{array}$ & $\begin{array}{c}\mathrm{Rs} \\
(\Omega)\end{array}$ \\
\hline ON & 0.25 & 38 & 0.25 & 38 & 0 & 10.2 & 27 & 0 \\
\hline OFF & 0.06 & 33 & 0.06 & 33 & 170 & 10.2 & 0 & 1.2 \\
\hline
\end{tabular}

FIGURE 9 The modeled data $v$ s. measurement data for a cantilever switch. 
conductor. To reduce the contact resistance, the contact area should be larger. $L_{S}$ is related to the inductance from the meandered cantilever beams. The isolation can be improved by providing a ground path in between the beams and the center conductor to achieve a wideband flat response.

The experiment results show that cantilever switch is capable of sub-10 volts operation. However, the reduced spring constant will undermine the switching speed as well. For a faster switching speed requirement and voltage operation requirement, the hinged switch may have the ability to meet these two requirements simultaneously.

\subsection{Hinged Switches}

Hinged switches have the potential to further reduce the actuation voltage of a MEM switch down to less than 5 volts. Shown in Figure 10 is a fabricated hinged switch. A suspended conductive pad with a width of $40 \mu \mathrm{m}$ and a length of $200 \mu \mathrm{m}$ hangs across the signal line and ground plane.

The measured data are shown in Figure 11. For the switch-on state (Fig. 11a), it shows an insertion loss of less than $0.1 \mathrm{~dB}$ with a return loss of better than $23 \mathrm{~dB}$ for frequencies up to $40 \mathrm{GHz}$. The insertion loss is a collective result of substrate radiation loss and the stray capacitance of the metal pad hanging on top of the CPW section. For the switch-off state (Fig. 11b), an isolation of higher than $25 \mathrm{~dB}$ across $40 \mathrm{GHz}$ bandwidth is achieved. The minimum switching voltage is 9 volts.

The extracted device parameters versus the measured data are shown in Figure 12. The smith chart shows that the equivalent models fit the measurement data very well up to frequency of $40 \mathrm{GHz}$. An off-state resistance of $1.2 \Omega$ and an on-state capacitance of $38 \mathrm{fF}$ are extracted from the equivalent circuit model. The parasitic capacitance is negligible, indicating the metal pad flatness is well controlled. A lower off-state resistance results in a

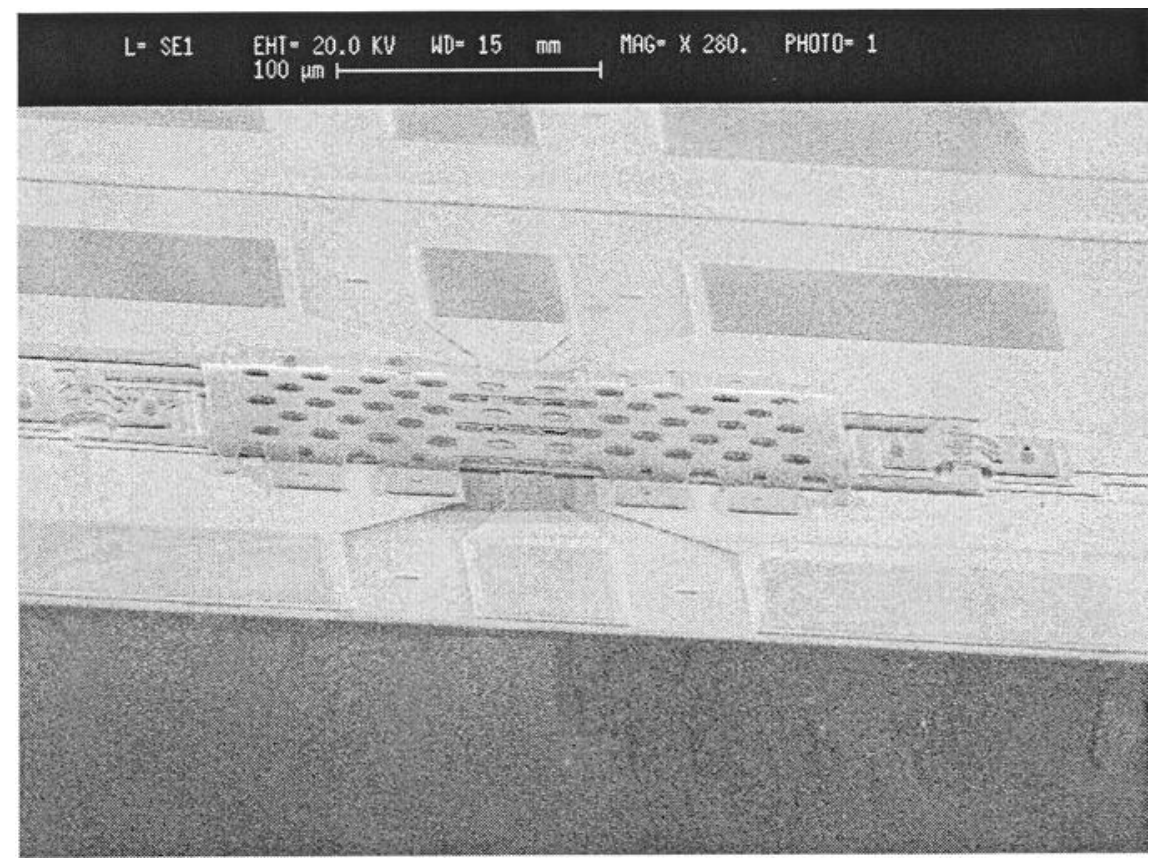

FIGURE 10 A SEM photograph of a fabricated hinged switch. 


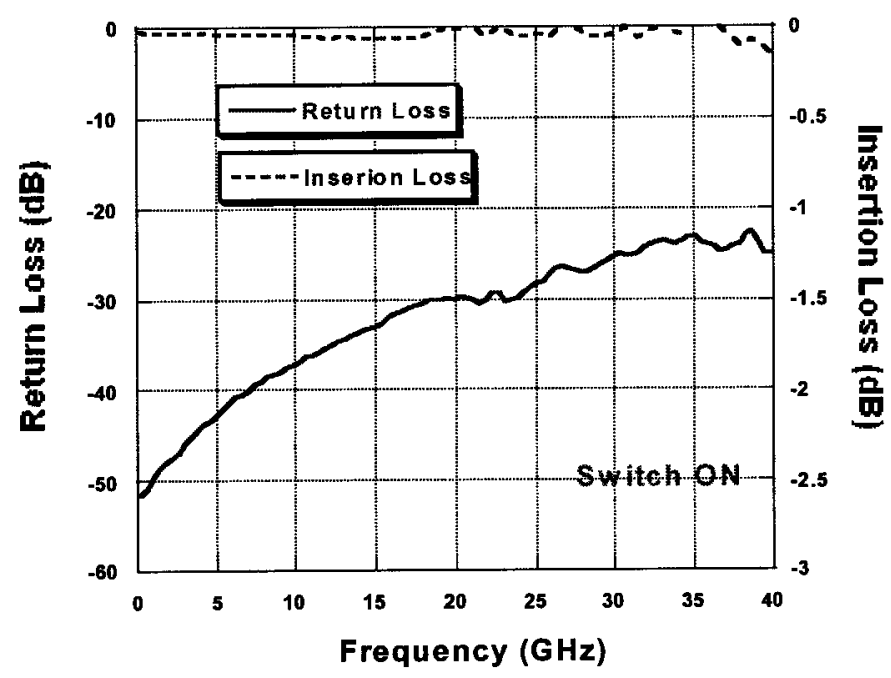

(a)

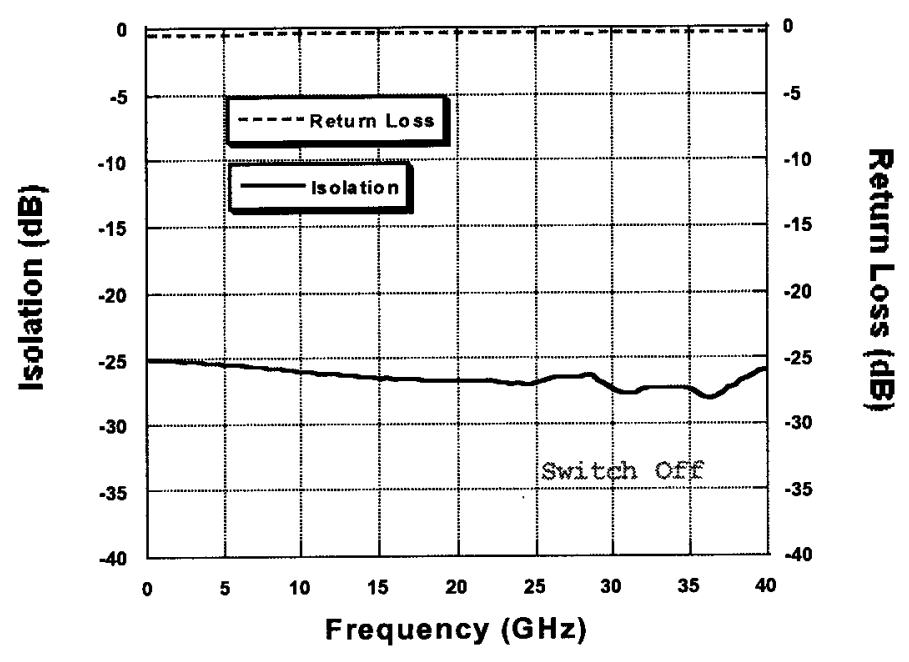

(b)

FIGURE 11 Measured data of hinged switch for (a) switch-on and (b) switch-off states.

higher isolation, and a smaller on-state capacitance indicates a lower insertion loss. The conductive pad size, therefore, becomes a trade-off parameter in hinged RF MEM switch design. The resulting device models show that the MEM switch is a very linear device for frequencies up to at least $40 \mathrm{GHz}$.

The flat frequency response for the switch-off state for both cantilever and hinged switches is due to the metal-to-metal contact between the center conductor and ground planes. The resistive coupling scheme results in a broadband switch compared with capacitively coupled shunt switches. The extremely broadband frequency response will lessen the complexity of 


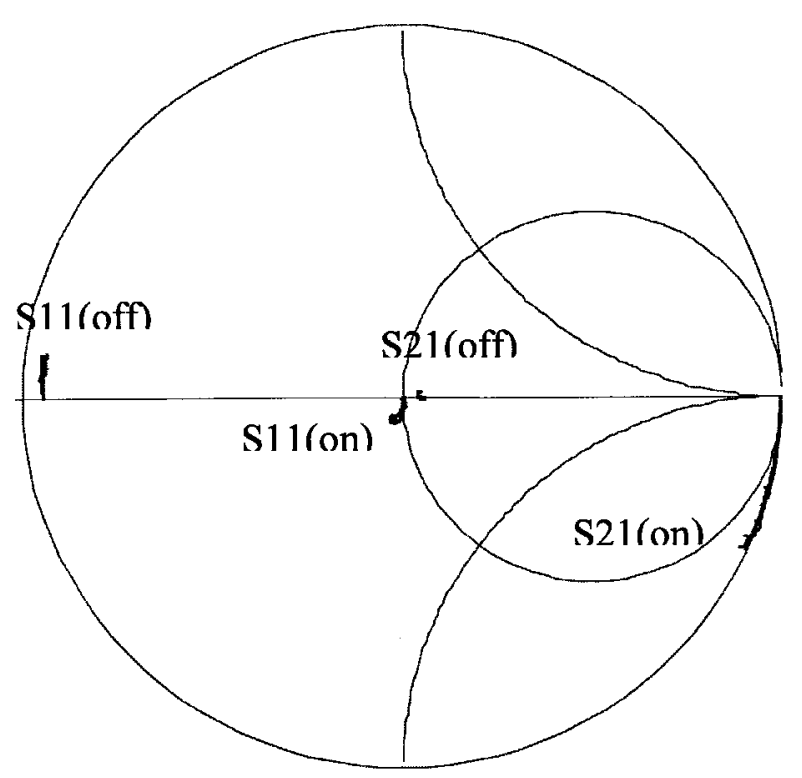

\begin{tabular}{|c|c|c|c|c|c|c|c|c|}
\hline State & $\begin{array}{c}\mathrm{R}_{\mathrm{L} 1} \\
(\Omega)\end{array}$ & $\begin{array}{c}\mathrm{L}_{\mathrm{L} 1} \\
(\mathrm{pH})\end{array}$ & $\begin{array}{c}\mathrm{R}_{\mathrm{L} 2} \\
(\Omega)\end{array}$ & $\begin{array}{c}\mathrm{L}_{\mathrm{L} 2} \\
(\mathrm{pH})\end{array}$ & $\begin{array}{c}\mathrm{C}_{\mathrm{p}} \\
(\mathrm{fF})\end{array}$ & $\begin{array}{c}\mathrm{L}_{\mathrm{s}} \\
(\mathrm{pH})\end{array}$ & $\begin{array}{c}\mathrm{C}_{\mathrm{s}} \\
(\mathrm{fF})\end{array}$ & $\begin{array}{c}\mathrm{Rs} \\
(\Omega)\end{array}$ \\
\hline ON & 0.26 & 37 & 0.26 & 37 & $\sim 0$ & 0 & 38 & 0 \\
\hline OFF & 0.23 & 13 & 0.23 & 13 & $\sim 0$ & 0 & 0 & 1.2 \\
\hline
\end{tabular}

FIGURE 12 The modeled data vs. measurement data for a hinged switch.

RF circuit design using RF MEM switches. The reduction in actuation voltage of the hinged switches is attributed to the proper control of metal pad warping and the use of the carbondioxide supercritical release processes.

\section{SUMMARY AND CONCLUSIONS}

In summary, we have demonstrated state-of-the-art sub-10 volts operation of RF MEM switches. Both cantilever switches and hinged switches are designed and fabricated. For cantilever switches, we demonstrate an operation voltage of less than 10 volts and a switching speed of $47 \mu$ s along with a wide band operation. A promising low actuation voltage of 9 volts is obtained for hinged switches with an isolation of better than $25 \mathrm{~dB}$ and an insertion loss of less than $0.1 \mathrm{~dB}$ over the frequency band of $40 \mathrm{GHz}$. Both of the switches are designed to achieve broadband RF characteristics. From experimental results, we found that cantilever switches suffer from their fundamental limitation, the spring constant. The operation voltage and switching speed become a trade-off of the switch performance. On the other hand, our proposed hinged switch can address these issues. We believe the hinged RF MEM switch will be a promising structure that will satisfy both low-voltage and high-speed requirement. The low-voltage switches will play an important role for the next generation MEMS-based wireless communication systems. 


\section{Acknowledgements}

This work is supported under the contract of DARPA-RECAP program: Mechanically Conformal and Electrically Reconfigurable APertureUsing Low Voltage MEMS and Flexible Membrane for Space Based Radar Applications - Contract No. : F33615-99-C-1519.

\section{References}

[1] Brown, E. R. (1998). RF_MEMS switches for reconfigurable integrated circuits. IEEE Trans. Microwave Theory and Tech., 46(11), 1868-1880.

[2] Larson, L. E., Hackett, R. H., Melendes, M. A. and Lohr, R. F. (1991). Micromachined microwave actuator (MIMAC) technology - a new tuning approach for microwave integrated circuits. In: IEEE Microwave Theory Tech. Symp., pp. 27-30.

[3] Yoon, J., Han, C., Yoon, E. and Kim, C. (1999). High performance three-dimensional on-chip inductors fabricated by novel micromachinging technology for RF MMIC. In: IEEE MTT-S International Microwave Symposium Digest.

[4] Feng, Z., Zhang, W., Su, B., Harsh, K., Gupta, K., Bright, V. and Lee, Y. (1999). Design and modeling of RF MEMS tunable capacitors using electro-thermal actuators. In: IEEE MTT-S International Microwave Symposium Digest.

[5] Zavracjy, P. M. and Morrison, R. H. (1984). Electrically actuated micromechanical switches with hysteresis. In: IEEE Solid State Sensor Conf.

[6] Yao, J. J. and Chang, M. F. (1995). A surface micromachined miniature switch for telecommunications applications with signal frequencies from DC up to $4 \mathrm{GHz}$. In: 8th International Conf. Solid-State Sens. and Actuators, Stockholm, Sweden, pp. 384-387.

[7] Goldsmith, C., Lin, T. H., Bill Powers, Wu, W. R. and Norvell, B. (1995). Micromechanical membrane switches for microwave applications. In: IEEE Microwave Theory Tech. Symp., pp. 91-94.

[8] Goldsmith, C., Randall, J., Eshelman, S., Lin, T. H., Denniston, D., Chen, S. and Norvell, B. (1996). Characteristics of micromachined switches at microwave frequencies. In: IEEE Microwave Theory Tech. Symp., vol. 2., pp. 1141-1144.

[9] Goldsmith, C. L., Yao, Z., Eshelman, S. and Denniston, D. (1998). Performance of low-loss RF MEMS capacitive switches. IEEE Microwave and Guided Wave Letters, 8(8), 269-271.

[10] Phillans, B., Eshelman, S., Malczewski, A., Ehmke, J. and Goldsmith, C. (1999). Ka-Band RF MEMS Phase Shifters. IEEE Microwave and Guided Wave Letters, 9(12), 520-522.

[11] Malczewski, A., Eshelaman, S., Pilklans, B., Ehmke, J. and Goldsmith, C. (1999). X-band RF MEMS phase shifters for phased array applications. IEEE Microwave and Guided Wave Lett., 9(12), 517-519.

[12] Hayden, J. S. and Rebeiz, G. M. (2000). One and two-bit low-loss cascadable MEMS distributed X-band phase shifters. In: IEEE MTT-S International Microwave Symposium Digest, pp. 161-164.

[13] Pacheco, S. P., Katehi, L. P. B. and Nguyen, C. T.-C. (2000). Design of low actuation voltage RF MEMS switch. In: IEEE MTT-S International Microwave Symposium Digest, pp. 165-168.

[14] Hah, D., Yoon, E., Hong, nad S. (2000). A low voltage actuated micromachined microwave switch using torsion spring and leverage. In: IEEE MTT-S International Microwave Symposium Digest, pp. 157-160.

[15] Barker, N. S. and Rebeiz, G. M. (1998). Distributed MEMS true-time delay phase shifters and wide-band switches. IEEE Trans. Microwave Theory and Tech., 46(11), 1881-1889.

[16] Feng, Milton and Shen, S. C. (2000). Low actuation voltage microelectromechanical device and method of manufacture, U.S. patent issued.

[17] Shen, S. C. and Feng, M. (1999). Low actuation voltage RF MEMS switches with signal frequency from $0.25 \mathrm{GHz}$ to $40 \mathrm{GHz}$. In: IEEE International Electron Device Meetings 1999, Dec. 5-8.

[18] Shen, S. C., Caruth, D. and Feng, M. (2000). Broadband low actuation voltage RF MEM switches. In: IEEE 2000 GaAs IC Symposium, Nov. 5-8 (to be presented).

[19] Pacheco, S., Nguyen, C. and Katehi, L. (1998). Micromechanical electrostatic Ka-Band switches. In: IEEE MTT$S$ Digest, pp. 1569-1572.

[20] Dyck, C. W., Smith, J. H., Miller, S. L., Russick, E. M., Adkins, C. L. J. (1996). Supercritical carbon dioxide solvent extraction from surface-micromachined micromechanical structures. SPIE Micromachining and Microfabrication, Oct.

[21] Caruth, D. C. (1999). Large signal modeling of MESFETs. MS Thesis, Univ. of Illinois, Urbana-Champaign.

[22] Shimon, R. (1998). The characteristic impedance and propagation constant of coplanar transmission lines. $M S$ Thesis, University of Illinois, Urbana Champaign. 

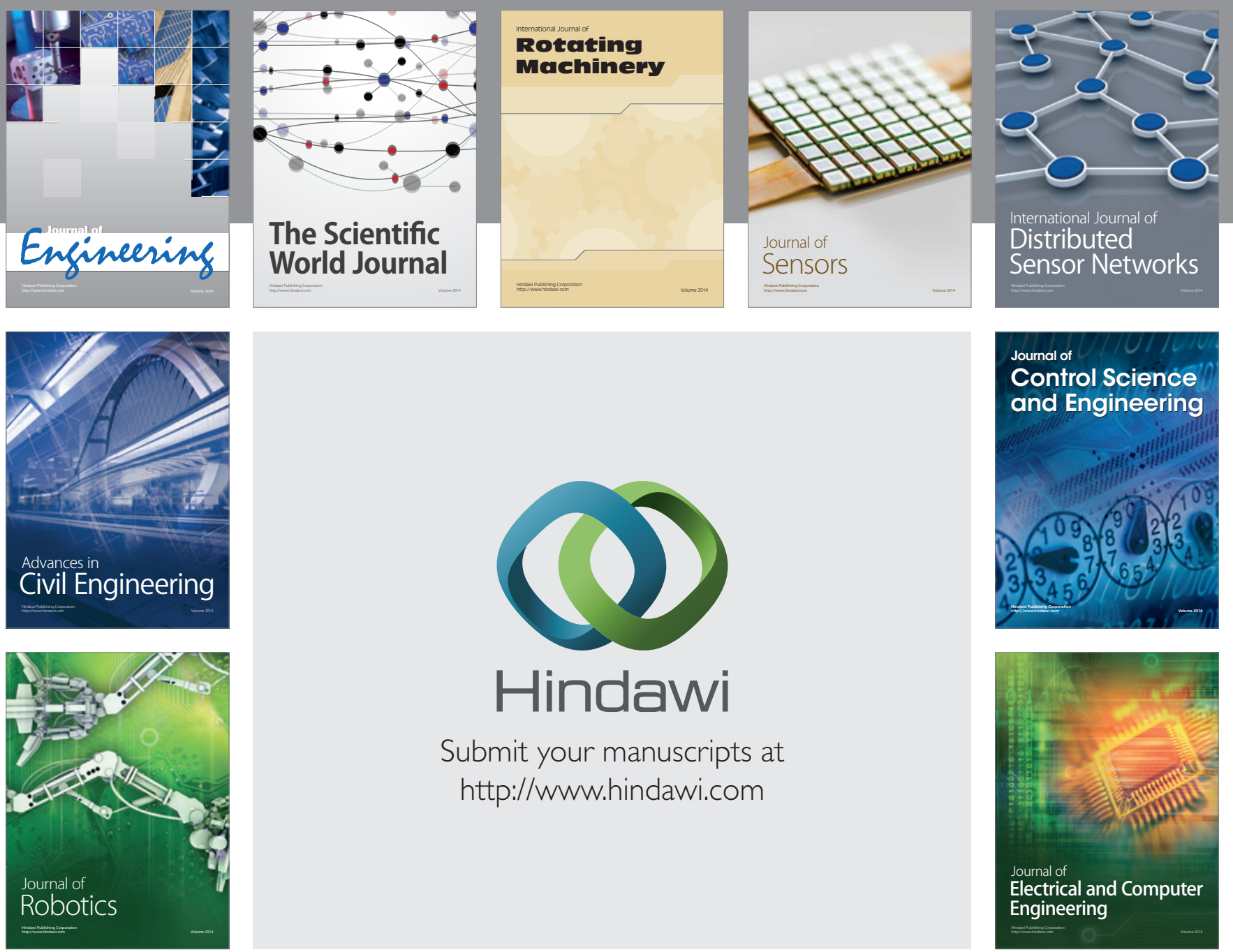

Submit your manuscripts at

http://www.hindawi.com
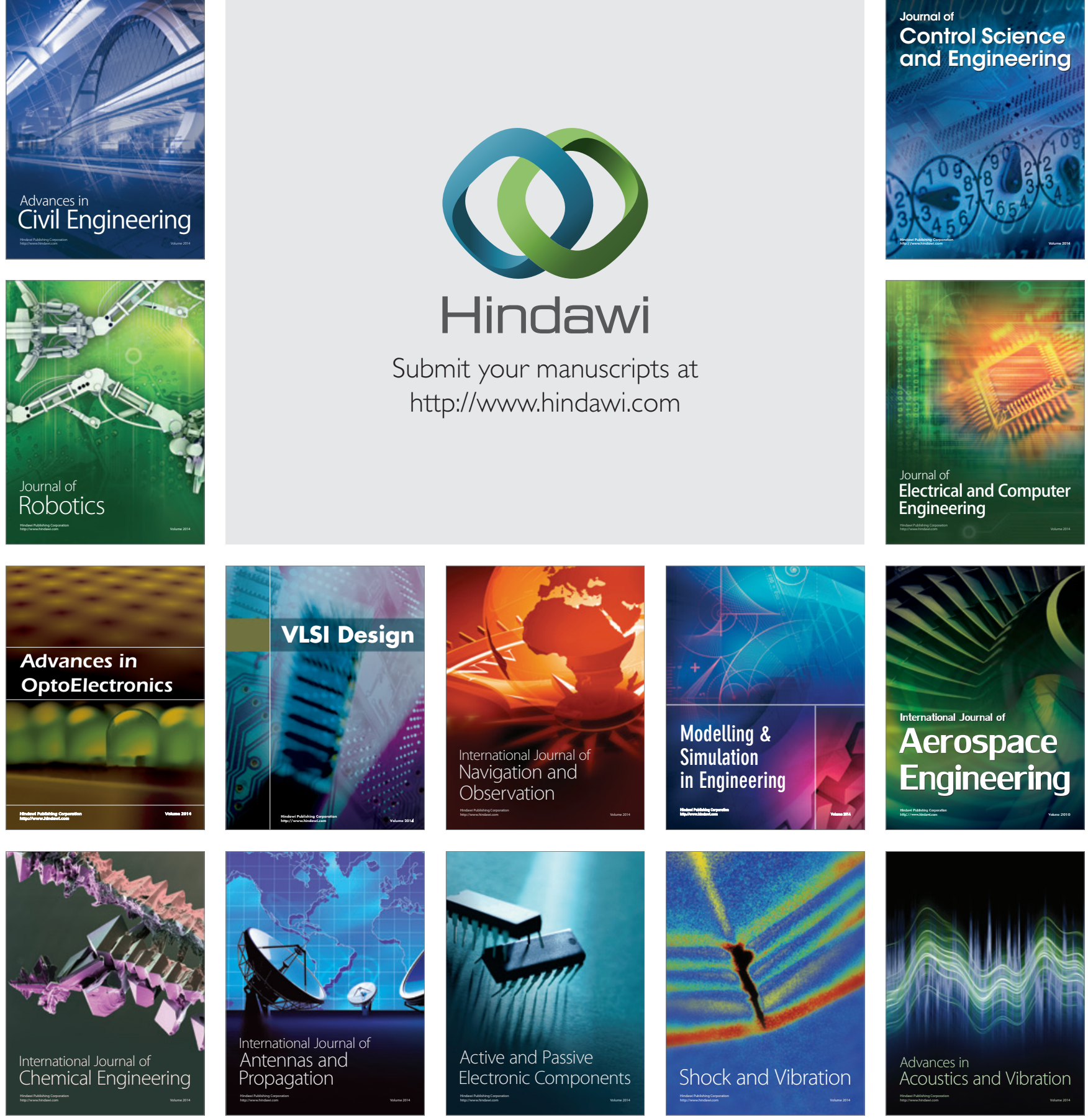